\title{
Converse Theorems for the DMC with
}

\section{Mismatched Decoding}

\author{
Anelia Somekh-Baruch
}

\begin{abstract}
The problem of mismatched decoding with an additive metric $q$ for a discrete memoryless channel $W$ is addressed. The "product-space" improvement of the random coding lower bound on the mismatch capacity, $C_{q}^{(\infty)}(W)$, was introduced by Csiszár and Narayan. It is equal to the supremum, as $k$ tends to infinity, of the rates that are achievable by random coding for the product channel $W^{k}$.

We study two kinds of decoders. The $\delta$-margin mismatched decoder outputs a message whose metric with the channel output exceeds that of all the other codewords by at least $\delta$. The $\tau$-threshold decoder outputs a single message whose metric with the channel output exceeds a threshold $\tau$. Both decoders declare an error if they fail to find a message that meets the requirement. It is assumed that $q$ is bounded.

It is proved that $C_{q}^{(\infty)}(W)$ is equal to the mismatch capacity with a constant margin decoder. We next consider sequences of $P$-constant composition codebooks, whose empirical distribution of the codewords are at least $o\left(n^{-1 / 2}\right)$ close in the $L_{1}$ distance sense to $P$. Using the Central Limit Theorem, it is shown that for such sequences of codebooks the supremum of achievable rates with constant threshold decoding is upper bounded by the supremum of the achievable rates with a constant margin decoder, and therefore also by $C_{q}^{(\infty)}(W)$.

Further, a soft converse is proved stating that if the average probability of error of a sequence of codebooks converges to zero sufficiently fast, the rate of the code sequence is upper bounded by $C_{q}^{(\infty)}(W)$. In particular, if $q$ is a bounded rational metric, and the average probability of error converges to zero faster than $O\left(n^{-1}\right)$, then $R \leq C_{q}^{(\infty)}(W)$. Finally, a max-min multi-letter upper bound on the mismatch capacity that bears some resemblance to $C_{q}^{(\infty)}(W)$ is presented.
\end{abstract}

A. Somekh-Baruch is with the Faculty of Engineering at Bar-Ilan University, Ramat-Gan, Israel. Email: somekha@biu.ac.il. This work was supported by the Israel Science Foundation (ISF) under grant 2013/919. Some of the results of this paper were presented at the IEEE International Symposium on Information Theory (ISIT) 2015 and at the 2016 International Zurich Seminar on Communications. This paper was accepted for publication in the IEEE Transactions on Information Theory. 


\section{INTRODUCTION}

Mismatched decoding is a prevalent channel coding paradigm in which the decoder has a fixed structure that cannot be tailored to the actual channel in use. The fixed structure of the decoder may be due to practical considerations such as decoding complexity or inaccurate channel estimation. It is usually assumed that among all codewords, the decoding rule maximizes a certain metric between the channel output sequence and the codeword. The encoder, who knows the metric used by the decoder, needs to construct an encoding scheme that will yield a vanishingly small average probability of error. The highest achievable rate using a given decoder metric is referred to as the mismatch capacity.

Mismatched decoding has been studied extensively, especially for discrete memoryless channels (DMCs). The formula for the achievable rates for the DMC with mismatched decoding using random coding, which is referred to as the LM rate, was derived by Csiszár and Körner [1] and by Hui [2]. In [1], an error exponent for random coding with fixed composition codes and mismatched decoding was established using a graph decomposition theorem. Lapidoth [3] introduced an improved lower bound on the mismatch capacity of the DMC by studying the achievable sumrate of an appropriately chosen multiple access channel (MAC) with mismatched decoding, whose codebook was obtained by expurgating codewords from the product of the codebooks of the two users. In [4], [5] the achievable region and error exponents of a cognitive MAC were considered using superposition coding or random binning whose sum-rate serves as a lower bound on the capacity of the single-user channel. An improved bound was presented by Scarlett et al. [6] (see also [7]) using a refinement of the superposition coding ensemble. For given auxiliary random variables, the results of [4]-[6] may yield improvement of the achievable rates of [3] for the DMC. For other related works and extensions see [7]-[17] and references therein.

In [18], the mismatch capacity of the DMC with a decoding metric $q$, denoted $C_{q}(W)$, was considered. It was shown that the LM rate is not tight in general but that its positivity is a necessary condition for positive mismatch capacity. This result was obtained by proving that the random coding bound for the product channel $W_{Y_{1}, \ldots, Y_{k} \mid X_{1}, \ldots, X_{k}}=\prod_{i=1}^{k} W_{Y_{i} \mid X_{i}}(k$ consecutive channel uses of the DMC $W)$, denoted $C_{q}^{(k)}(W)$, could result in strictly higher achievable rates. They referred to the improved bound as the "product-space" improvement of the lower bound, and the supremum of the achievable rates obtained by taking the limit of $C_{q}^{(k)}(W)$ as $k$ tends to infinity was denoted $C_{q}^{(\infty)}(W)$.

In the special case of erasures-only (e.o.) capacity, the product space improvement $C_{q}^{(\infty)}(W)$ was shown to be tight. Csiszár and Narayan conjectured that the mismatch capacity is indeed given by $C_{q}^{(\infty)}(W)$. Whether this conjecture is true in general remains an open question.

It was further stated in [18] that "although the bound is not computable, its tightness would afford some valuable conclusions, for instance, that for $R<C_{q}(W)$, codes with $d$-decoding always exist with rates approaching $R$ and probability of error approaching zero exponentially fast". Another implication of an affirmative answer to the conjecture concerns the threshold capacity of the DMC. The threshold capacity is the supremum of achievable rates obtained by decoding the unique message which accumulates a metric that exceeds a predetermined threshold. It was stated in [18] that if the conjecture is true, the threshold capacity and the mismatch capacity of the DMC are 
equal.

Unlike lower bounds, upper bounds on the mismatch capacity have only been provided in some special cases. The only non-trivial single-letter converse result reported in [19] for binary-input DMCs was recently disproved in [20]. Specifically, a rate based on superposition coding was shown to exceed the claimed mismatch capacity of [19].

In [21] a general formula was derived in the form of a Verdú-Han [22] expression for the mismatch capacity of a general channel, and was defined as a sequence of conditional distributions with a general decoding metric sequence. Further, several upper bounds on the capacity were provided, and a simpler expression for a lower bound was derived for the case of a non-negative decoding metric. The general formula was specialized to the case of finite input and output alphabet channels with a type-dependent metric. The problem of threshold mismatched decoding was also studied, and a general expression for the threshold mismatch capacity was obtained.

In this paper we focus on a DMC with mismatched decoding with an additive metric, and study several properties of the "product-space" improvement of the random coding lower bound on the mismatch capacity, $C_{q}^{(\infty)}(W)$. We define a mismatched decoder with a $\delta$-margin as a decoder that decides in favor of the message whose metric with the channel output exceeds that of all the other codewords by at least $\delta$ : if no such prominent codeword exists, an error is declared. The mismatch capacity with a $\delta$-margin is the supremum of achievable rates with mismatched $\delta$-margin decoders. The mismatch capacity with a constant margin decoder is the supremum over $\delta>0$ of the mismatch capacity with a $\delta$-margin. The word constant is used to emphasize the fact that the margin level $\delta$ is kept constant for all block lengths. Note that maximum likelihood (ML) decoding with a $\delta_{n}$-margin was studied by Lapidoth and Ziv [23, Equation (17)], where it was called a threshold decoder (see also [24]). Nevertheless, since threshold decoding usually refers to a decoder that outputs the single codeword whose metric exceeds a certain threshold, we chose to adopt the term "margin decoder" as stated above to distinguish between the two terms.

The first result presented in this paper is a proof that $C_{q}^{(\infty)}(W)$ is equal to the mismatch capacity with a constant margin decoder. The significance of the result stems from the fact that if we can prove that the capacity with a constant margin decoder of the DMC is equal to the mismatch capacity, it could be inferred that the mismatch capacity is equal to $C_{q}^{(\infty)}(W)$. We also introduce the notion of constant threshold decoding, which is the supremum of achievable rates with threshold decoding with a threshold level that is kept constant for all block lengths.

We establish an inequality between the supremum of achievable rates using constant margin decoding and constant threshold decoding, under the assumption that the sequence of codebooks is $P$-constant composition (a term that will be defined rigorously). We further prove a soft converse stating that if the minimum probability of error achievable for block-length $n$ converges to zero sufficiently fast, the rate of the code is upper bounded by $C_{q}^{(\infty)}(W)$. In particular, if $q$ is a bounded rational metric, and the lowest achievable average probability of error, $\epsilon_{n}$, satisfies $\lim _{n \rightarrow \infty} n \epsilon_{n}=0$, then $R \leq C_{q}^{(\infty)}(W)$. Since for any $k$, at rates below $C_{q}^{(k)}(W)$ there exist code sequences with an average probability of error that vanishes exponentially fast, this implies that $C_{q}^{(\infty)}(W)$ is the supremum of the rates below which there exist codes with an average probability of error that vanishes exponentially fast.

We further present an upper bound on the transmission rate: a max-min multi-letter upper bound on the mismatch capacity $C_{q}(W)$ is derived. This upper bound can be thought of as the product space improvement of the random 
coding lower bound with a genie-aided decoder that is informed of the joint empirical statistics of the output symbols $y_{i}, i=1 \ldots, n$ and their metrics with respect to the channel inputs, $q\left(x_{i}, y_{i}\right), i=1, \ldots, n$. Some of the results of this paper were presented in [25].

The remainder of this paper is as follows. In Section [I] we present some notational conventions. Section [II provides a formal statement of the communication channel setup and definitions. In Section IV an equality between $C_{q}^{(\infty)}(W)$ and the capacity with a constant margin decoder is established, a relationship between the achievable rate of a constant margin decoder and a constant threshold decoder is introduced, and $C_{q}^{(\infty)}(W)$ is characterized as the highest achievable average probability of error which decays sufficiently fast. Section $\nabla$ is devoted to deriving a max-min upper bound on the mismatch capacity of the DMC. The discussion and concluding remarks appear in Section VI

\section{NOTATION}

Throughout this paper, scalar random variables are denoted by capital letters, their sample values are denoted by their respective lower case letters, and their alphabets are denoted by their respective calligraphic letters, e.g. $X$, $x$, and $\mathcal{X}$, respectively. A similar convention applies to random vectors of dimension $n$ and their sample values, which are superscripted by $n$, e.g.., $x^{n}$. The set of all $n$-vectors with components taking values in a certain finite alphabet are denoted by the same alphabet superscripted by n, e.g., $\mathcal{X}^{n}$. Logarithms are taken to the natural base $e$.

For a given sequence $\boldsymbol{y} \in \mathcal{Y}^{n}$, where $\mathcal{Y}$ is a finite alphabet, $\hat{P} \boldsymbol{y}$ denotes the empirical distribution on $\mathcal{Y}$ extracted from $\boldsymbol{y}$; in other words, $\hat{P}_{\boldsymbol{y}}$ is the vector $\left\{\hat{P}_{\boldsymbol{y}}(y), y \in \mathcal{Y}\right\}$, where $\hat{P}_{\boldsymbol{y}}(y)$ is the relative frequency of the symbol $y$ in the vector $\boldsymbol{y}$. The type-class of $\boldsymbol{x}$ is the set of $\boldsymbol{x}^{\prime} \in \mathcal{X}^{n}$ such that $\hat{P}_{\boldsymbol{x}^{\prime}}=\hat{P}_{\boldsymbol{x}}$, which is denoted $T\left(\hat{P}_{\boldsymbol{x}}\right)$. Let $\mathcal{P}(\mathcal{X})$ denote the set of all probability distributions on $\mathcal{X}$, The set of empirical distributions of order $n$ on alphabet $\mathcal{X}$ is denoted $\mathcal{P}_{n}(\mathcal{X})$.

Information theoretic quantities such as entropy, conditional entropy, and mutual information are denoted following the usual conventions in the information theory literature, e.g., $H(X), H(X \mid Y), I(X ; Y)$ and so on. To emphasize the dependence of the quantity on a certain underlying probability distribution, say $\mu$, it is subscripted by $\mu$, e.g., with notations such as $H_{\mu}(X), H_{\mu}(X \mid Y), I_{\mu}(X ; Y)$, etc. The expectation operator is denoted by $\mathbb{E}(\cdot)$, and once again, to make the dependence on the underlying distribution $\mu$ clear, it is denoted by $\mathbb{E}_{\mu}(\cdot)$. The cardinality of a finite set $\mathcal{A}$ is denoted by $|\mathcal{A}|$. The indicator function of an event $\mathcal{E}$ is denoted by $1\{\mathcal{E}\}$.

\section{PRELIMINARIES}

Consider a DMC with a finite input alphabet $\mathcal{X}$ and a finite output alphabet $\mathcal{Y}$, which is governed by the conditional p.m.f. $W$. As the channel is fed by an input vector $x^{n} \in \mathcal{X}^{n}$, it generates an output vector $y^{n} \in \mathcal{Y}^{n}$ according to the sequence of conditional probability distributions

$$
P\left(y_{i} \mid x_{1}, \ldots, x_{i}, y_{1}, \ldots, y_{i-1}\right)=W\left(y_{i} \mid x_{i}\right), \quad i=1,2, \ldots, n
$$

where for $i=1,\left(y_{1}, \ldots, y_{i-1}\right)$ is understood as the null string. 
A rate- $R$ block-code of length $n$ consists of $e^{n R} n$-vectors $x^{n}(m), m=1,2, \ldots, e^{n R}$, which represent $e^{n R}$ different messages; i.e., it is defined by the encoding function

$$
f_{n}:\left\{1, \ldots, e^{n R}\right\} \rightarrow \mathcal{X}^{n}
$$

It is assumed that all the possible messages are a-priori equiprobable; i.e., $P(m)=e^{-n R}$ for all $m \in\left\{1, \ldots, e^{n R}\right\}$. A mismatched decoder for the channel is defined by a mapping

$$
q_{n}: \mathcal{X}^{n} \times \mathcal{Y}^{n} \rightarrow \mathbb{R}
$$

where the decoder declares that message $i$ was transmitted iff

$$
q_{n}\left(x^{n}(i), y^{n}\right)>q_{n}\left(x^{n}(j), y^{n}\right), \forall j \neq i
$$

and if no such $i$ exists, an error is declared.

An important class of mismatched decoders is the class of additive decoding functions; i.e.,

$$
q_{n}\left(x^{n}, y^{n}\right)=\frac{1}{n} \sum_{i=1}^{n} q\left(x_{i}, y_{i}\right)
$$

where $q$ is a mapping from $\mathcal{X} \times \mathcal{Y}$ to $\mathbb{R}$.

The results that will be presented in this paper hold under one of the following boundedness assumptions (to be specified when needed).

The first assumption is that $q$ is bounded as follows: there exists $B \geq 0$ such that

$$
|q(x, y)| \leq B<\infty, \forall(x, y) \in \mathcal{X} \times \mathcal{Y}: W(y \mid x)>0
$$

The second assumption is slightly more restrictive: there exists $B \geq 0$ such that

$$
q(x, y) \leq B<\infty, \forall(x, y) \in \mathcal{X} \times \mathcal{Y} \text { and } q(x, y) \geq-B>-\infty, \forall(x, y) \in \mathcal{X} \times \mathcal{Y}: W(y \mid x)>0
$$

Note that the matched metric $q(x, y)=\log W(y \mid x)$ satisfies both assumptions (6) and (7).

Below are several useful definitions which refer to a general channel $W^{(n)}$ from $\mathcal{X}^{n}$ to $\mathcal{Y}^{n}$ and to the DMC $W^{(n)}=W^{n}$ as a special case. It is useful to define the average probability of error associated with a codebook, a channel and a metric:

Definition 1. For a given codebook $\mathcal{C}_{n}$, let $P_{e}\left(W^{(n)}, \mathcal{C}_{n}, q_{n}\right)$ designate the average probability of error incurred by the decoder $q_{n}$ (4) employed on the output of the channel $W^{(n)}$.

Definition 2. A code $\mathcal{C}_{n}$ with a decoding metric $q_{n}$ is an $(n, M, \epsilon)$-code for the channel $W^{(n)}$ if it has $M$ codewords of length $n$ and $P_{e}\left(W^{(n)}, \mathcal{C}_{n}, q_{n}\right) \leq \epsilon$.

We next define an $\epsilon$-achievable rate and the mismatch capacity.

Definition 3. A rate $R>0$ is an $\epsilon$-achievable rate for the channel $\boldsymbol{W}=\left\{W^{(n)}\right\}_{n \in \mathbb{N}}$ with decoding metric sequence $\boldsymbol{q}=\left\{q_{n}\right\}_{n \in \mathbb{N}}$ if for every $\delta>0$, there exists a sequence of codes $\left\{\mathcal{C}_{n}\right\}_{n \in \mathbb{N}}$ such that for all $n$ sufficiently large, $\mathcal{C}_{n}$ is an $\left(n, M_{n}, \epsilon\right)$ code for the channel $W^{(n)}$ and decoding metric $q_{n}$ with rate $\frac{\log \left(M_{n}\right)}{n} \geq R-\delta$. 
Definition 4. The mismatch capacity of channel $W$ with an additive decoding metric $q$, denoted $C_{q}(W)$, is the supremum of rates that are $\epsilon$-achievable for all $0<\epsilon<1$.

As mentioned in the introduction, it was proved in [1], [2] that the mismatch capacity of the DMC is lower bounded by the LM rate given by the formula

$$
C_{q}^{(1)}(W)=\max _{P_{X}} \min _{P_{\tilde{Y} \mid X}: P_{\tilde{Y}}=P_{Y}, \mathbb{E}(q(X, \widetilde{Y})) \geq \mathbb{E}(q(X, Y))} I(X ; \tilde{Y}),
$$

where $P_{X} \in \mathcal{P}(\mathcal{X}),(X, Y) \sim P_{X} \times W,(X, \widetilde{Y}) \sim P_{X} \times P_{\widetilde{Y} \mid X}$, and $P_{Y}, P_{\widetilde{Y}}$ are the corresponding marginal distributions of $Y, \tilde{Y}$, respectively. By considering the achievable rate for channel $W^{k}$ from $\mathcal{X}^{k}$ to $\mathcal{Y}^{k}$, the following rate is also achievable $([18])$

$$
C_{q}^{(k)}(W)=\max _{P_{X^{k}}} \min _{P_{\tilde{Y}^{k} \mid X^{k}}: P_{\widetilde{Y}^{k}}=P_{Y^{k}}, \mathbb{E}\left(q_{k}\left(X^{k}, \widetilde{Y}^{k}\right)\right) \geq \mathbb{E}\left(q_{k}\left(X^{k}, Y^{k}\right)\right)} \frac{1}{k} I\left(X^{k} ; \widetilde{Y}^{k}\right),
$$

where $P_{X^{k}} \in \mathcal{P}\left(\mathcal{X}^{k}\right),\left(X^{k}, Y^{k}\right) \sim P_{X^{k}} \times W^{k},\left(X^{k}, \widetilde{Y}^{k}\right) \sim P_{X^{k}} \times P_{\widetilde{Y}^{k} \mid X^{k}}$, and $P_{Y^{k}}, P_{\widetilde{Y}^{k}}$ are the corresponding marginal distributions of $Y^{k}, \widetilde{Y}^{k}$, respectively. Since for all $k$ the rate $C_{q}^{(k)}(W)$ is achievable, then so is

$$
C_{q}^{(\infty)}(W)=\limsup _{k \rightarrow \infty} C_{q}^{(k)}(W) .
$$

A closely related notion to that of a mismatched decoder is the $q_{n}$-decoder with a $\delta$-margin, which decides that $i$ is the transmitted message iff

$$
q_{n}\left(x^{n}(i), y^{n}\right) \geq q_{n}\left(x^{n}(j), y^{n}\right)+\delta, \forall j \neq i,
$$

and if no such $i$ exists an error is declared.

The following definition extends $P_{e}\left(W^{(n)}, \mathcal{C}_{n}, q_{n}\right)$ to the case of a margin decoder.

Definition 5. For a given codebook $\mathcal{C}_{n}$, let $P_{\text {margin }}\left(W^{(n)}, \mathcal{C}_{n}, q_{n}, \delta\right)$ designate the average probability of error incurred by the $q_{n}$ decoder with a $\delta$-margin (11) employed on the output of the channel $W^{(n)}$.

An $(n, M, \epsilon)$-code and an $\epsilon$-achievable rate with respect to $\delta$-margin decoding are defined similarly to Definitions 2 and 3 . The mismatch capacity using the additive decoding metric $q_{n}(5)$ with a $\delta$-margin is denoted $C_{q, \text { margin }}(W, \delta)$. Finally, we define the mismatch capacity with a constant margin decoder as

$$
C_{q, \text { margin }}^{\text {const }}(W) \triangleq \sup _{\delta>0} C_{q, \text { margin }}(W, \delta) .
$$

It is easy to see that $C_{q, \text { margin }}^{\text {const }}(W) \leq C_{q}(W)$ since the margin decoder is more restrictive and if the $q_{n}$ decoder errs, the $q_{n}$ decoder with a $\delta$-margin errs as well.

Another decoding rule that is related to mismatched $q_{n}$-decoder is the $\left(q_{n}, \tau\right)$-threshold decoder which decides that $i$ is the transmitted message iff

$$
q_{n}\left(x^{n}(i), y^{n}\right) \geq \tau
$$

and

$$
q_{n}\left(x^{n}(j), y^{n}\right)<\tau, \forall j \neq i
$$


The following definition extends $P_{e}\left(W^{(n)}, \mathcal{C}_{n}, q_{n}\right)$ to the case of a threshold decoder,

Definition 6. For a given codebook $\mathcal{C}_{n}$, let $P_{\text {thresh }}\left(W^{(n)}, \mathcal{C}_{n},\left(q_{n}, \tau\right)\right)$ designate the average probability of error incurred by the $\left(q_{n}, \tau\right)$-threshold decoder (13)-(14) employed on the output of channel $W^{(n)}$.

An $(n, M, \epsilon)$-code and an $\epsilon$-achievable rate with respect to $\tau$-threshold decoding are defined similarly to Definitions 2 and 3

Definition 7. The constant threshold q-capacity of a channel denoted $C_{q, \text { threshold }}^{\text {const }}(W)$ is defined as the supremum over $\tau$ of the rates achievable by codes with $\left(q_{n}, \tau\right)$-threshold decoders.

One has $C_{q, \text { threshold }}^{\text {const }}(W) \leq C_{q}(W)$, since a threshold decoder (13)-(14) is more restrictive than the mismatched decoder (4).

\section{Properties of $C_{q}^{(\infty)}(W)$}

In this section we prove several properties of $C_{q}^{(\infty)}(W)$. The first property is that $C_{q}^{(\infty)}(W)$ is equal to $C_{q, \text { margin }}^{\text {const }}(W)$ and the second is that $C_{q}^{(\infty)}(W)$ is the highest achievable rate for an average probability of error $P_{e}\left(W^{(n)}, \mathcal{C}_{n}, q_{n}\right)$ which vanishes sufficiently fast. Further, we consider sequences of $P$-constant composition codebooks, such that the empirical distribution of the codewords converges to $P$ (at least $o\left(n^{-1 / 2}\right)$-fast). It is shown that the supremum of the achievable rates with constant threshold decoding for such sequences of codebooks is upper bounded by the supremum of the achievable rates with a constant margin decoder, and therefore also by $C_{q}^{(\infty)}(W)$

Before proving these properties we recall that the dual expression for the $n$-letter product-form expression of the $\mathrm{LM}$ rate ([10], [12], [13]) in the case of an additive metric can be expressed as

$$
C_{q}^{(n)}(W)=\frac{1}{n} \max _{P^{(n)} \in \mathcal{P}\left(\mathcal{X}^{n}\right)} \sup _{s_{n} \geq 0, a\left(x^{n}\right) \geq 0} \mathbb{E}\left(\log \frac{e^{s_{n} q_{n}\left(X^{n}, Y^{n}\right)+a\left(X^{n}\right)}}{\sum_{\bar{x}^{n}} P^{(n)}\left(\bar{x}^{n}\right) e^{s_{n} q_{n}\left(\bar{x}^{n}, Y^{n}\right)+a\left(\bar{x}^{n}\right)}}\right),
$$

where $\left(X^{n}, Y^{n}\right) \sim P^{(n)} \times W^{n}$. The following lemma will be useful in lower bounding $C_{q}^{(n)}(W)$ and therefore also $C_{q}^{(\infty)}(W)$.

Lemma 1. Let $q$ be bounded as in (7), let $\mathcal{A} \subseteq \mathcal{X}^{n} \times \mathcal{Y}^{n}$, fix $s_{n} \geq 0$, and fix a subset $\mathcal{C}_{n} \subseteq \mathcal{X}^{n}$ of size $\left|\mathcal{C}_{n}\right|=e^{n R_{n}}$. Let $P^{(n)}$ denote the uniform distribution over $\mathcal{C}_{n}$, and let $\left(X^{n}, Y^{n}\right) \sim P^{(n)} \times W^{n}$, then

$$
\begin{aligned}
& \sup _{a\left(x^{n}\right) \geq 0} \mathbb{E}\left(\log \frac{e^{s_{n} q_{n}\left(X^{n}, Y^{n}\right)+a\left(X^{n}\right)}}{\sum_{\bar{x}^{n}} P^{(n)}\left(\bar{x}^{n}\right) e^{s_{n} q_{n}\left(\bar{x}^{n}, Y^{n}\right)+a\left(\bar{x}^{n}\right)}}\right) \\
& \geq-\operatorname{Pr}\{\mathcal{A}\} \cdot \frac{s_{n} \cdot 2 B}{n} \\
& \quad+\operatorname{Pr}\left\{\mathcal{A}^{c}\right\} \cdot \mathbb{E}\left(\min \left\{R_{n}, \frac{s_{n}}{n} \cdot \min _{\bar{x}^{n} \in \mathcal{C}_{n}: \bar{x}^{n} \neq X^{n}}\left[q_{n}\left(X^{n}, Y^{n}\right)-q_{n}\left(\bar{x}^{n}, Y^{n}\right)\right]\right\} \mid \mathcal{A}^{c}\right)-\frac{1}{n} \log (2),
\end{aligned}
$$

where $\operatorname{Pr}\{\mathcal{A}\}=\operatorname{Pr}\left\{\left(X^{n}, Y^{n}\right) \in \mathcal{A}\right\}$, and $B$ is the metric bound in (7).

Lemma 1 will be used in the proofs of Theorems 1 and 3 , with different substitutions for the event $\mathcal{A}$. In Theorem 1, $\mathcal{A}^{c}$ will stand for the event of $\delta$-margin correct decoding, under which the term 
$\min _{\bar{x}^{n} \in \mathcal{C}_{n}: \bar{x}^{n} \neq X^{n}}\left[q_{n}\left(X^{n}, Y^{n}\right)-q_{n}\left(\bar{x}^{n}, Y^{n}\right)\right]$ exceeds $\delta$. For Theorem 3 , $\mathcal{A}^{c}$ will signify the event of correct mismatched decoding, under which the same term can be lower bounded by the minimum value of $q_{n}\left(x^{n}, y^{n}\right)-$ $q_{n}\left(\bar{x}^{n}, y^{n}\right)$ among all possible triplets of sequences $\left(x^{n}, \bar{x}^{n}, y^{n}\right)$.

We next prove Lemma 1

Proof. First note that choosing $a\left(x^{n}\right)=0$ for all $x^{n}$ rather than taking the supermum over $a\left(x^{n}\right) \geq 0$, we obtain the lower bound

$$
\begin{aligned}
& \sup _{a\left(x^{n}\right) \geq 0} \mathbb{E}\left(\log \frac{e^{s_{n} q_{n}\left(X^{n}, Y^{n}\right)+a\left(X^{n}\right)}}{\sum_{\bar{x}^{n}} P^{(n)}\left(\bar{x}^{n}\right) e^{s_{n} q_{n}\left(\bar{x}^{n}, Y^{n}\right)+a\left(\bar{x}^{n}\right)}}\right) \\
\geq & \frac{1}{n} \mathbb{E}\left(\log \frac{e^{s_{n} q_{n}\left(X^{n}, Y^{n}\right)}}{\sum_{\bar{x}^{n}} P^{(n)}\left(\bar{x}^{n}\right) e^{s_{n} q_{n}\left(\bar{x}^{n}, Y^{n}\right)}}\right) \\
= & \operatorname{Pr}\{\mathcal{A}\} \cdot \frac{1}{n} \mathbb{E}\left(\log \frac{e^{s_{n} q_{n}\left(X^{n}, Y^{n}\right)}}{\sum_{\bar{x}^{n}} P^{(n)}\left(\bar{x}^{n}\right) e^{s_{n} q_{n}\left(\bar{x}^{n}, Y^{n}\right)}} \mid \mathcal{A}\right)+\operatorname{Pr}\left\{\mathcal{A}^{c}\right\} \cdot \frac{1}{n} \mathbb{E}\left(\log \frac{e^{s_{n} q_{n}\left(X^{n}, Y^{n}\right)}}{\sum_{\bar{x}^{n}} P^{(n)}\left(\bar{x}^{n}\right) e^{s_{n} q_{n}\left(\bar{x}^{n}, Y^{n}\right)}} \mid \mathcal{A}^{c}\right),
\end{aligned}
$$

where the last step follows from the law of total probability. Now, define

$$
\begin{aligned}
& q_{\max }\left(Y^{n}\right) \triangleq \max _{x^{n} \in \mathcal{C}_{n}} q_{n}\left(x^{n}, Y^{n}\right), \\
& Z_{n} \triangleq q_{\max }\left(Y^{n}\right)-q_{n}\left(X^{n}, Y^{n}\right)
\end{aligned}
$$

and note that the boundedness assumption (7) implies that $Z_{n} \leq 2 B$. Therefore,

$$
\log \frac{e^{s_{n} q_{n}\left(X^{n}, Y^{n}\right)}}{\sum_{\bar{x}^{n}} P^{(n)}\left(\bar{x}^{n}\right) e^{s_{n} q_{n}\left(\bar{x}^{n}, Y^{n}\right)}} \geq \log \frac{e^{s_{n} q_{n}\left(X^{n}, Y^{n}\right)}}{e^{s_{n} q_{\max }\left(Y^{n}\right)}}=s_{n}\left(q_{n}\left(X^{n}, Y^{n}\right)-q_{\max }\left(Y^{n}\right)\right) \geq-s_{n} \cdot 2 B,
$$

and consequently we can lower bound the first conditional expectation in (17) as follows

$$
\mathbb{E}\left(\frac{1}{n} \log \frac{e^{s_{n} q_{n}\left(X^{n}, Y^{n}\right)}}{\sum_{\bar{x}^{n}} P^{(n)}\left(\bar{x}^{n}\right) e^{s_{n} q_{n}\left(\bar{x}^{n}, Y^{n}\right)}} \mid \mathcal{A}\right) \geq-\frac{s_{n} \cdot 2 B}{n} .
$$

As for the second conditional expectation in 177,

$$
\begin{aligned}
& \mathbb{E}\left(\frac{1}{n} \log \frac{e^{s_{n} q_{n}\left(X^{n}, Y^{n}\right)}}{\sum_{\bar{x}^{n}} P^{(n)}\left(\bar{x}^{n}\right) e^{s_{n} q_{n}\left(\bar{x}^{n}, Y^{n}\right)}} \mid \mathcal{A}^{c}\right) \\
& =-\mathbb{E}\left(\frac{1}{n} \log \left(P^{(n)}\left(X^{n}\right)+\sum_{\bar{x}^{n} \in \mathcal{C}_{n}: \bar{x}^{n} \neq X^{n}} P^{(n)}\left(\bar{x}^{n}\right) e^{-s_{n}\left[q_{n}\left(\bar{x}^{n}, Y^{n}\right)-q_{n}\left(X^{n}, Y^{n}\right)\right]}\right) \mid \mathcal{A}^{c}\right) \\
& \stackrel{(*)}{=}-\mathbb{E}\left(\frac{1}{n} \log \left(e^{-n R_{n}}+\sum_{\bar{x}^{n} \in \mathcal{C}_{n}: \bar{x}^{n} \neq X^{n}} P^{(n)}\left(\bar{x}^{n}\right) e^{-s_{n}\left[q_{n}\left(\bar{x}^{n}, Y^{n}\right)-q_{n}\left(X^{n}, Y^{n}\right)\right]}\right) \mid \mathcal{A}^{c}\right)
\end{aligned}
$$

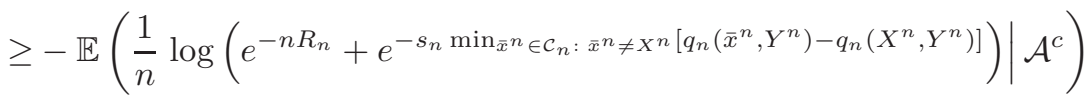

$$
\begin{aligned}
& \geq \mathbb{E}\left(\min \left\{R_{n}, \frac{s_{n}}{n} \cdot \min _{\bar{x}^{n} \in \mathcal{C}_{n}: \bar{x}^{n} \neq X^{n}}\left[q_{n}\left(X^{n}, Y^{n}\right)-q_{n}\left(\bar{x}^{n}, Y^{n}\right)\right]\right\} \mid \mathcal{A}^{c}\right)-\frac{1}{n} \log (2),
\end{aligned}
$$

where $(*)$ follows since $P^{(n)}$ is uniform over $\mathcal{C}_{n}$, and the last step follows since $e^{-a}+e^{-b} \leq 2 e^{-\min \{a, b\}}$. 


\section{A. An Equality Between $C_{q}^{(\infty)}$ and the Capacity with Margin Decoding}

The next theorem states that $C_{q}^{(\infty)}$ is equal to $C_{q, \text { margin }}^{\text {const }}(W)$, and implies that the following two statements are equivalent: (a) $C_{q}(W)=C_{q}^{(\infty)}(W)$ and (b) $C_{q}(W)=C_{q, \text { margin }}^{\text {const }}(W)$.

Theorem 1. For a metric $q$ which satisfies the boundedness assumption (7) one has

$$
C_{q}^{(\infty)}(W)=C_{q, \text { margin }}^{\text {const }}(W)
$$

The theorem is established by proving the two inequalities, $C_{q}^{(\infty)}(W) \geq C_{q, \text { margin }}^{\text {const }}(W)$ and $C_{q}^{(\infty)}(W) \leq$ $C_{q, m a r g i n}^{\text {const }}(W)$. The proof of the latter is based on Lemma $\square$ where the event $\mathcal{A}$ in 16 is chosen as the error event in margin decoding and with a proper choice of parameter $s_{n}$.

Proof. Proof of $C_{q}^{(\infty)}(W) \leq C_{q, \text { margin }}^{\text {const }}(W)$ :

The average probability of error associated with a random coding constant composition scheme within the typeclass $T\left(P_{X}\right)$, and a $\delta$-margin decoder can be expressed as

$$
\bar{P}_{e}=\sum_{x^{n} \in T\left(P_{X}\right), y^{n}} \frac{1}{\left|T\left(P_{X}\right)\right|} W^{n}\left(y^{n} \mid x^{n}\right)\left[1-\left(1-\sum_{\bar{x}^{n} \in T\left(P_{X}\right): q_{n}\left(\bar{x}^{n}, y^{n}\right) \geq q_{n}\left(x^{n}, y^{n}\right)-\delta} \frac{1}{\left|T\left(P_{X}\right)\right|}\right)^{M_{n}}\right],
$$

where $1-\bar{P}_{e}$ stands for the probability of drawing a transmitted codeword whose metric with the channel output exceeds that of all the other independently drawn codewords by at least $\delta$. Optimizing $P_{X}$ and using standard method of types tools and the weak law of large numbers, one can deduce that for all $\epsilon>0$, the rate $C_{q, \text { margin }}^{(1)}(W, \delta)-\epsilon$ is achievable with a $\delta$-margin decoder, where

$$
C_{q, \text { margin }}^{(1)}(W, \delta)=\max _{P_{X}} \min _{P_{\tilde{Y} \mid X}: P_{\tilde{Y}}=P_{Y}, \mathbb{E}(q(X, \widetilde{Y})) \geq \mathbb{E}(q(X, Y))-\delta} I(X ; \widetilde{Y}),
$$

with $P_{X} \in \mathcal{P}(\mathcal{X}),(X, Y) \sim P_{X} \times W,(X, \widetilde{Y}) \sim P_{X} \times P_{\widetilde{Y} \mid X}$, and where $P_{Y}, P_{\widetilde{Y}}$ are the corresponding marginal distributions of $Y, \widetilde{Y}$, respectively. Now, using the continuity of $C_{q, \text { margin }}^{(1)}(W, \delta)$ w.r.t. $\delta$ (which follows from its convexity, see e.g., [18, Appendix VI]), we obtain $\lim _{\delta \downarrow 0} C_{q, \operatorname{margin}}^{(1)}(W, \delta)=C_{q}^{(1)}(W)$, and as a result, the $\delta$-margin capacity is lower bounded by $C_{q}^{(1)}(W)$ and therefore also by $C_{q}^{(\infty)}(W)$. Consequently we obtain $C_{q, \text { margin }}^{\text {const }}(W)=$ $\sup _{\delta>0} C_{q, \operatorname{margin}}(W, \delta) \geq C_{q}^{(\infty)}(W)$.

$\underline{\text { Proof of } C_{q}^{(\infty)}(W) \geq C_{q, \text { margin }}^{\text {const }}(W)}$ :

Fix $\delta$ and a vanishing sequence $\epsilon_{n}, n \geq 1$. Let $\mathcal{P}_{U}\left(\epsilon_{n}, \delta\right)$ stand for the set of $P^{(n)} \in \mathcal{P}\left(\mathcal{X}^{n}\right)$ which are uniform over a subset $\mathcal{C}_{n}$ of $\mathcal{X}^{n}$ and such that $P_{\text {margin }}\left(W^{(n)}, \mathcal{C}_{n}, q_{n}, \delta\right)=\epsilon_{n}$; i.e.,

$$
\begin{aligned}
& \mathcal{P}_{U}\left(\epsilon_{n}, \delta\right) \\
\triangleq & \left\{P^{(n)} \in \mathcal{P}\left(\mathcal{X}^{n}\right): P^{(n)}\left(\tilde{x}^{n}\right)=P^{(n)}\left(x^{n}\right) \text { if } P^{(n)}\left(x^{n}\right) \cdot P^{(n)}\left(\tilde{x}^{n}\right)>0, P_{\text {margin }}\left(W^{(n)}, \mathcal{C}_{n}, q_{n}, \delta\right)=\epsilon_{n}\right\},
\end{aligned}
$$

where $\mathcal{C}_{n}$ stands for the support of $P^{(n)}$. 
Since $\mathcal{P}_{U}\left(\epsilon_{n}, \delta\right) \subseteq \mathcal{P}\left(\mathcal{X}^{n}\right)$,

$$
C_{q}^{(n)}(W) \geq \frac{1}{n} \max _{P^{(n)} \in \mathcal{P}_{U}\left(\epsilon_{n}, \delta\right)} \sup _{s_{n} \geq 0} \mathbb{E}\left(\log \frac{e^{s_{n} q_{n}\left(X^{n}, Y^{n}\right)}}{\sum_{\bar{x}^{n}} P^{(n)}\left(\bar{x}^{n}\right) e^{s_{n} q_{n}\left(\bar{x}^{n}, Y^{n}\right)}}\right) .
$$

Let $P^{(n)} \in \mathcal{P}_{U}\left(\epsilon_{n}, \delta\right)$ be given, and denote its support by $\mathcal{C}_{n}$. Next, let $\mathcal{E}$ denote the error event

$$
\mathcal{E}=\left\{\left(x^{n}, y^{n}\right): q_{n}\left(x^{n}, y^{n}\right)<\max _{\tilde{x}^{n} \in \mathcal{C}_{n}: \tilde{x}^{n} \neq x^{n}} q_{n}\left(\tilde{x}^{n}, y^{n}\right)+\delta\right\}
$$

and note that $\operatorname{Pr}(\mathcal{E})=P_{\text {margin }}\left(W^{(n)}, \mathcal{C}_{n}, q_{n}, \delta\right)=\epsilon_{n}$.

Now recall (15), we obtain for $X^{n} \sim P^{(n)}$ that is uniform over a subset $\mathcal{C}_{n} \in \mathcal{X}^{n}$ of size $\left|\mathcal{C}_{n}\right|=e^{R_{n}}$,

$$
\begin{aligned}
& C_{q}^{(n)}(W) \\
= & \frac{1}{n} \sup _{s_{n} \geq 0, a\left(x^{n}\right) \geq 0} \mathbb{E}\left(\log \frac{e^{s_{n} q_{n}\left(X^{n}, Y^{n}\right)+a\left(X^{n}\right)}}{\sum_{\bar{x}^{n}} P^{(n)}\left(\bar{x}^{n}\right) e^{s_{n} q_{n}\left(\bar{x}^{n}, Y^{n}\right)+a\left(\bar{x}^{n}\right)}}\right) \\
\geq & \sup _{s_{n} \geq 0}-\operatorname{Pr}\{\mathcal{E}\} \cdot \frac{s_{n} \cdot 2 B}{n}+\operatorname{Pr}\left\{\mathcal{E}^{c}\right\} \cdot \mathbb{E}\left(\min \left\{R_{n}, \frac{s_{n}}{n} \cdot \min _{\bar{x}^{n} \in \mathcal{C}_{n}: \bar{x}^{n} \neq X^{n}}\left[q_{n}\left(X^{n}, Y^{n}\right)-q_{n}\left(\bar{x}^{n}, Y^{n}\right)\right]\right\} \mid \mathcal{E}^{c}\right) \\
& \quad-\frac{1}{n} \log (2) \\
\geq & \sup _{s_{n} \geq 0}-\frac{\epsilon_{n} s_{n} \cdot 2 B}{n}+\left(1-\epsilon_{n}\right) \cdot \min \left\{R_{n}, \frac{s_{n} \delta}{n}\right\}-\frac{1}{n} \log 2,
\end{aligned}
$$

where the first inequality follows from (16) by substituting $\mathcal{A} \Rightarrow \mathcal{E}$ and the second in equality follows since under $\mathcal{E}^{c}$, we have $\min _{\bar{x}^{n} \in \mathcal{C}_{n}: \bar{x}^{n} \neq X^{n}}\left[q_{n}\left(X^{n}, Y^{n}\right)-q_{n}\left(\bar{x}^{n}, Y^{n}\right)\right] \geq \delta$.

Taking $1 s_{n}=\frac{n R_{n}}{\delta}$, it follows that

$$
C_{q}^{(n)}(W) \geq \max _{P^{(n)} \in \mathcal{P}_{U}\left(\epsilon_{n}, \delta\right)}-\frac{\epsilon_{n} \cdot 2 B R_{n}}{\delta}+\left(1-\epsilon_{n}\right) \cdot R_{n}-\frac{1}{n} \log 2 .
$$

Now, since this inequality holds for every $\delta>0$ and every non-negative vanishing sequence $\epsilon_{n}, n \geq 1$, we obtain

$$
\begin{aligned}
C_{q}^{(\infty)}(W) & \geq \sup _{\substack{\delta>0,\left\{\epsilon_{n}\right\}_{n \geq 1}: \epsilon_{n} \geq 0, \lim _{n \rightarrow \infty} \epsilon_{n}=0}} \limsup _{n \rightarrow \infty} \max _{P^{(n)} \in \mathcal{P}_{U}\left(\epsilon_{n}, \delta\right)}-\frac{\epsilon_{n} \cdot 2 B R_{n}}{\delta}+\left(1-\epsilon_{n}\right) R_{n} \\
& =C_{q, \text { margin }}^{\text {const }}(W),
\end{aligned}
$$

where last step follows since the supremum of $R$ over $\delta$ such that there exists a sequence of codebooks $\mathcal{C}_{n}, n \geq 1$ with $\frac{1}{n} \log \left|\mathcal{C}_{n}\right| \geq R$ and which incur $\lim _{n \rightarrow \infty} \epsilon_{n}=0$ is nothing but $C_{q, \text { margin }}^{\text {const }}(W)$. This concludes the proof of the inequality $C_{q}^{(\infty)}(W) \geq C_{q, \text { margin }}^{\text {const }}(W)$ and Theorem 1 follows.

A special case of a decoding metric is the erasures-only metric

$$
q_{\text {e.o. }}(x, y)=1\{W(y \mid x)>0\} .
$$

This metric has the property that no undetected errors can occur, since by definition the transmitted codeword always accumulates a 1-value metric, which is the highest possible. Since in the erasures-only metric case, it has been shown ([18]) that the mismatch capacity is equal to the threshold capacity and to $C_{q}^{(\infty)}(W)$, we deduce from Theorem 1 that in this case the mismatch capacity with a constant margin decoder is equal to the mismatch capacity.

\footnotetext{
${ }^{1}$ We choose $s_{n}=\frac{n R_{n}}{\delta}$ to balance the effect of the two terms $-\frac{\epsilon_{n} s_{n} \cdot 2 B}{n}$ and $\min \left\{R_{n}, \frac{s_{n} \delta}{n}\right\}$, and to obtain an asymptotic lower bound of $R_{n}$. Any choice smaller than $n R_{n} / \delta$ would result in the second term being strictly smaller than $R_{n}$.
} 
Note that the proof of Theorem 1 implies that taking $a\left(x^{n}\right)=0$ in 15 and maximizing over $P^{(n)}$ incurs a loss of rate (compared to the optimal value of non-constant $a\left(x^{n}\right)$ ) which vanishes as $n$ tends to infinity.

To see this, let

$$
\widetilde{C}_{q}^{(n)}(W)=\frac{1}{n} \max _{P^{(n)} \in \mathcal{P}\left(\mathcal{X}^{n}\right)} \sup _{s_{n} \geq 0} \mathbb{E}\left(\log \frac{e^{s_{n} q_{n}\left(X^{n}, Y^{n}\right)}}{\sum_{\bar{x}^{n}} P^{(n)}\left(\bar{x}^{n}\right) e^{\left.s_{n} q_{n}\left(\bar{x}^{n}, Y^{n}\right)\right)}}\right),
$$

and observe that the first step (17) of the proof is to lower bound $C_{q}^{(n)}(W)$ by $\widetilde{C}_{q}^{(n)}(W)$, so the same proof actually gives:

$$
C_{q}^{(\infty)}(W) \geq \widetilde{C}_{q}^{(\infty)}(W) \geq C_{q, \text { margin }}^{\text {const }}(W)
$$

On the other hand, it is also proven that $C_{q}^{(\infty)}(W) \leq C_{q, \operatorname{margin}}^{\text {const }}(W)$ so we have:

$$
C_{q}^{(\infty)}(W) \geq \widetilde{C}_{q}^{(\infty)}(W) \geq C_{q, \text { margin }}^{\text {const }}(W) \geq C_{q}^{(\infty)}(W)
$$

This sandwich argument shows that $C_{q}^{(\infty)}(W)=\widetilde{C}_{q}^{(\infty)}(W)$. Indeed, for finite $n$ there is usually a strict inequality $\widetilde{C}_{q}^{(n)}(W)<C_{q}^{(n)}(W)$, as it is known that the generalized mutual information (GMI) [10] can be strictly smaller than the LM rate. Nevertheless, in the limit as $n$ tends to infinity, they are equal. The explanation we provide is that without loss of asymptotic optimality the maximizing $P^{(n)}$ (in the dual expression (15) can be replaced by a uniform distribution over a subset of a single type-class in $\mathcal{X}^{n}$, denoted $\mathcal{C}$. Asymptotically, this may cancel the need to assign different $a(\cdot)$ values (in the dual expression (15) ) to different sequences $x^{n}$, at least in the bounded metric case.

\section{B. A Relationship Between Constant Margin decoding and Constant Threshold Decoding}

In this section we establish a connection between the capacity with constant threshold decoding and decoding with a constant margin decoder.

Definition 8. Let $P \in \mathcal{P}(\mathcal{X})$ be given. A sequence of constant-composition codebooks $\mathcal{C}_{n}, n=1,2, \ldots$ is said to be P-constant composition, if for all $n$, the codewords lie in a single type-class which corresponds to an empirical distribution $\hat{P}_{n}$ that is $o\left(n^{-1 / 2}\right)$ close in the $L_{1}$ distance sense to $P$; i.e.,

$$
\limsup _{n \rightarrow \infty} \frac{\left\|\hat{P}_{n}-P\right\|_{1}}{n^{-1 / 2}}=\limsup _{n \rightarrow \infty} \frac{\sum_{x \in \mathcal{X}}\left|\hat{P}_{n}(x)-P(x)\right|}{n^{-1 / 2}}=0 .
$$

We next define the constant threshold capacity and the constant margin capacity which are constrained to sequences of $P$-constant composition codes.

Definition 9. The $(P, q)$-threshold capacity, denoted $C_{q, \text { thresh }}^{\text {const }}(P, W)$ is the supremum of achievable rates using sequences of P-constant composition codes, metic q, and a constant threshold decoder.

Definition 10. The $(P, q)$-margin capacity, denoted $C_{q, \text { margin }}^{c o n s t}(P, W)$ is the supremum of achievable rates using sequences of P-constant composition codes, metic q, and a constant margin decoder. 
Theorem 2. If $P \in \mathcal{P}(\mathcal{X})$ is such that $\forall x \in \mathcal{X}, P(x)>0, q$ is bounded (6), and there exists at least one symbol

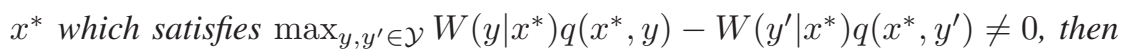

$$
C_{q, \text { threshold }}^{\text {const }}(P, W) \leq C_{q, \text { margin }}^{\text {const }}(P, W) \leq C_{q}^{(\infty)}(W) .
$$

Note that the more general opposite inequality $C_{q, \text { threshold }}^{\text {const }}(W) \geq C_{q}^{(\infty)}(W)$ has been known [18] and is proved similarly to the proof of the claim $C_{q}^{(\infty)}(W) \leq C_{q, \text { margin }}^{\text {const }}(W)$ (replacing $q_{n}\left(x^{n}, y^{n}\right)$ in 24 by $\left.\mathbb{E}(q(X, Y))-\tau\right)$.

It would have been desirable to answer the more general question whether $C_{q, \text { thresh }}^{\text {const }}(W) \leq C_{q}^{(\infty)}(W)$ or not. Clearly, this result would follow if $C_{q, \text { thresh }}^{\text {const }}(W)=\sup _{P \in \mathcal{P}(\mathcal{X}): \forall x \in \mathcal{X}, P(x)>0} C_{q, \text { threshold }}^{\text {const }}(P, W)$. It is important to note that the requirement that the sequence of codebooks be constant-composition does not limit the generality 2 . The requirement that might limit the generalization of the result is (36); that the empirical distribution converges to some $P$ at a rate which is $o\left(n^{-1 / 2}\right)$. The mismatch capacity as well as the $\delta$-margin capacity are insensitive to small fluctuations in the type class in which the codewords lie. This is because the decision regions and the corresponding error probabilities dictated by (4) and (11) do not change if each codeword of a codebook is concatenated by a constant identical string of symbols of length $f_{n}$ where $\lim _{n \rightarrow \infty} f_{n} / n=0$, this simply adds the same quantity to both sides of the equations (4) and (11). The threshold capacity with a given threshold sequence $\tau_{n}$ is more sensitive to such fluctuations, but we conjecture that this issue is minor and does not have a significant effect on the generality of the results.

The requirements on $P$ in Theorem $\Omega$ are needed to invoke the Central Limit Theorem (CLT) which gives a strictly positive non-vanishing lower bound on $\operatorname{Pr}\left\{q_{n}\left(X^{n}, Y^{n}\right)<\mathbb{E}_{\hat{P}_{n} \times W}(q(X, Y))-k n^{-1 / 2}\right\}$. This forces the threshold decoder to use a threshold level that is lower than $\mathbb{E}_{\hat{P}_{n} \times W}(q(X, Y))-k n^{-1 / 2}$.

As mentioned above, if $q=q_{\text {e.o }}$ the theorem holds, hence we can assume $q \neq q_{\text {e.o }}$. The condition that there exists at least one symbol $x^{*}$ which satisfies $\max _{y, y^{\prime} \in \mathcal{Y}} W\left(y \mid x^{*}\right) q\left(x^{*}, y\right)-W\left(y^{\prime} \mid x^{*}\right) q\left(x^{*}, y^{\prime}\right) \neq 0$ is not restrictive because it is not hard to realize that the result $C_{q}^{(\infty)}(W)=C_{q}(W)$ for $q=q_{e . o}$ ([18]) extends to the case of a metric which satisfies $\max _{y, y^{\prime} \in \mathcal{Y}}\left|W(y \mid x) q(x, y)-W\left(y^{\prime} \mid x\right) q\left(x, y^{\prime}\right)\right|=0, \forall x$ as well for constant composition codes.

We now prove Theorem 2 ,

Proof. The right-most inequality in (37) is a direct consequence of Theorem 11 The rest of the proof relies on the following two lemmas.

Lemma 2. Let $P \in \mathcal{P}(\mathcal{X})$ which satisfies the conditions of Theorem 2 and a sequence $\mathcal{C}_{n}, n=1,2, \ldots$ of $P$-constant composition codes be given where $\mathcal{C}_{n} \subseteq T\left(\hat{P}_{n}\right)$. Let $X^{n}$ denote the random codeword which is uniformly distributed over $\mathcal{C}_{n}$, and let $Y^{n}$ be the corresponding output of the channel $W$, then

$$
\liminf _{n \rightarrow \infty} \operatorname{Pr}\left\{q_{n}\left(X^{n}, Y^{n}\right)<\mathbb{E}_{\hat{P}_{n} \times W}(q(X, Y))-k n^{-1 / 2}\right\}>0, \forall 0<k<\infty .
$$

The proof of Lemma 2 is based on the CLT and appears in Appendix A

\footnotetext{
${ }^{2}$ The generality is not limited since the number of type-classes grows polynomially with $n$, whereas the number of codewords grows exponentially with $n$.
} 
Lemma 3. Let $X^{n}, Y^{n}$ be defined as in Lemma 2 then for all $\epsilon>0$,

$$
\begin{aligned}
& P_{\text {margin }}\left(W^{(n)}, \mathcal{C}_{n}, q_{n}, \epsilon\right) \\
\leq & P_{\text {thresh }}\left(W^{(n)}, \mathcal{C}_{n},\left(q_{n}, \tau\right)\right)+\operatorname{Pr}\left\{q_{n}\left(X^{n}, Y^{n}\right) \leq \tau+\epsilon\right\} .
\end{aligned}
$$

The proof of Lemma 3 appears in Appendix B,

Equipped with the lemmas we can now prove the theorem. From the condition 36 and since $\mid \mathbb{E}_{\hat{P}_{n} \times W}(q(X, Y))-$ $\mathbb{E}_{P \times W}(q(X, Y)) \mid \leq\left(q_{\max }-q_{\min }\right) \cdot\left\|\hat{P}_{n}-P\right\|_{1}$, where $q_{\min }=\min _{x, y: W(y \mid x)>0} q(x, y), q_{\max }=$ $\max _{x, y: W(y \mid x)>0} q(x, y)$, we have for all $n$ sufficiently large,

$$
\left|\mathbb{E}_{P \times W}(q(X, Y))-\mathbb{E}_{\hat{P}_{n} \times W}(q(X, Y))\right| \leq\left(q_{\max }-q_{\min }\right) n^{-1 / 2} .
$$

Therefore, for all $n$ sufficiently large,

$$
\begin{aligned}
& \operatorname{Pr}\left\{q_{n}\left(X^{n}, Y^{n}\right)<\mathbb{E}_{P \times W}(q(X, Y))\right\} \\
\geq & \operatorname{Pr}\left\{q_{n}\left(X^{n}, Y^{n}\right)<\mathbb{E}_{\hat{P}_{n} \times W}(q(X, Y))-\left(q_{\text {max }}-q_{\text {min }}\right) n^{-1 / 2}\right\} .
\end{aligned}
$$

We conclude from (38) that

$$
\begin{aligned}
& \liminf _{n \rightarrow \infty} \operatorname{Pr}\left\{q_{n}\left(X^{n}, Y^{n}\right)<\mathbb{E}_{P \times W}(q(X, Y))\right\} \\
\geq & \liminf _{n \rightarrow \infty} \operatorname{Pr}\left\{q_{n}\left(X^{n}, Y^{n}\right)<\mathbb{E}_{\hat{P}_{n} \times W}(q(X, Y))-\left(q_{\max }-q_{\text {min }}\right) n^{-1 / 2}\right\} \\
> & 0 .
\end{aligned}
$$

Since $\liminf \operatorname{in}_{n \rightarrow \infty} \operatorname{Pr}\left\{q_{n}\left(X^{n}, Y^{n}\right)<\tau\right\}=0$ is a necessary condition for a vanishing probability of error in $\tau$ threshold decoding, we deduce that for a sequence of $P$-constant composition codebooks, the constant threshold level $\tau$ should be strictly lower than $\mathbb{E}_{P \times W}(q(X, Y))$. Hence, denote $\tau=\mathbb{E}_{P \times W}(q(X, Y))-2 \epsilon$ where $\epsilon>0$, thus from 39 we obtain

$$
\begin{aligned}
& P_{\text {margin }}\left(W^{(n)}, \mathcal{C}_{n}, q_{n}, \epsilon\right) \\
\leq & P_{\text {thresh }}\left(W^{(n)}, \mathcal{C}_{n},\left(q_{n}, \tau\right)\right)+\operatorname{Pr}\left\{q_{n}\left(X^{n}, Y^{n}\right) \leq \mathbb{E}_{P \times W}(q(X, Y))-\epsilon\right\} .
\end{aligned}
$$

We will next show that the right most term $\operatorname{Pr}\left\{q_{n}\left(X^{n}, Y^{n}\right) \leq \mathbb{E}_{P \times W}(q(X, Y))-\epsilon\right\}$ vanishes as $n$ tends to infinity, which will imply that if $P_{\text {thresh }}\left(W^{(n)}, \mathcal{C}_{n},\left(q_{n}, \tau\right)\right)$ vanishes then so does $P_{\operatorname{margin}}\left(W^{(n)}, \mathcal{C}_{n}, q_{n}, \epsilon\right)$.

Define

$$
\begin{aligned}
& n_{x}=\sum_{i=1}^{n} 1\left\{X_{i}=x\right\} \\
& A_{x}=\frac{1}{n_{x}} \sum_{i=1}^{n} 1\left\{X_{i}=x\right\} \cdot q\left(x, Y_{i}\right)
\end{aligned}
$$

and note that

$$
q_{n}\left(X^{n}, Y^{n}\right)=\sum_{x=1}^{|\mathcal{X}|} \frac{n_{x}}{n} A_{x},
$$

and that $n_{x}$ is a deterministic quantity since all $x^{n}$,s lie in the same type-class. 
By assumption, $P(x)>0, \forall x \in \mathcal{X}$, from (36) we have that there exists $\zeta>0$ such that $A_{x}$ is a average of at least $n \zeta$ non deterministic i.i.d. random variables. This implies that $q_{n}\left(X^{n}, Y^{n}\right)$ is the weighted average of $|\mathcal{X}|$ independent random variables $A_{x}, x \in \mathcal{X}$, each of which is the average of at least $n \cdot \zeta$ non deterministic i.i.d, random variables. Thus, from (36) we obtain for sufficiently large $n$,

$$
\begin{aligned}
& \operatorname{Pr}\left\{q_{n}\left(X^{n}, Y^{n}\right) \leq \mathbb{E}_{P \times W}(q(X, Y))-\epsilon\right\} \\
& \stackrel{(a)}{\leq} \operatorname{Pr}\left\{q_{n}\left(X^{n}, Y^{n}\right) \leq \mathbb{E}_{\hat{P}_{n} \times W}(q(X, Y))-\epsilon / 2\right\} \\
& \stackrel{(b)}{\leq} \operatorname{Pr}\left\{\cup_{x=1}^{|\mathcal{X}|}\left\{A_{x} \leq \mathbb{E}_{W(\cdot \mid x)}(q(x, Y))-\epsilon / 2\right\}\right\} \\
& \leq \sum_{x=1}^{|\mathcal{X}|} \operatorname{Pr}\left\{A_{x} \leq \mathbb{E}_{W(\cdot \mid x)}(q(x, Y))-\epsilon / 2\right\},
\end{aligned}
$$

where (a) follows from (40), (b) follows from (45) and since for a p.m.f. $\mu \in \mathcal{P}(\mathcal{X})$, if $\sum_{x=1}^{|\mathcal{X}|} \mu(x) a_{x} \leq$ $\sum_{x=1}^{|\mathcal{X}|} \mu(x) b_{x}$, then there must exist at least one $x$ such that $a_{x} \leq b_{x}$, and the last step follows from the union bound. From the weak law of large numbers we have that the r.h.s. of (46) vanishes as $n$ tends to infinity. Thus, from (43) it follows that if $P_{\text {thresh }}\left(W^{(n)}, \mathcal{C}_{n},\left(q_{n}, \tau\right)\right)$ vanishes as $n$ tends to infinity, then so does $P_{\text {margin }}\left(W^{(n)}, \mathcal{C}_{n}, q_{n}, \epsilon\right)$. Consequently, under the assumptions of Theorem 2 the supremum of the achievable rates with a constant margin decoder is at least as high as that of a constant threshold decoder and Theorem 2 follows.

Note that in fact the proof of Theorem 1 implies a stronger result, that is stated in the following corollary.

Corollary 1. Let $\delta_{n}$ be a vanishing sequence which satisfies $\lim _{n \rightarrow \infty} \delta_{n} n^{1 / 2}=\infty$. If $P$ satisfies the conditions of Theorem 2 then for all P-constant composition sequences of codebooks, the supremum of the achievables rates with a $\delta_{n}$-margin decoder is at least as high as that of any time varying $\tau_{n}$ threshold decoder.

Proof. The proof goes along the lines of the proof of Theorem 2 where in (43) one can substitute $\epsilon$ with a sequence $\delta_{n}$ which vanishes more slowly than $O\left(n^{-1 / 2}\right)$. This is because in fact

$$
\liminf _{n \rightarrow \infty} \frac{\tau_{n}-\left(\mathbb{E}_{P \times W}(q(X, Y))-n^{-1 / 2}\right)}{n^{-1 / 2}}<0
$$

should hold, so without loss of generality one can set $\tau_{n}=\mathbb{E}_{P \times W}\left(q(X, Y)-2 \delta_{n}\right.$ and then from the CLT, we shall still have that the r.h.s. of (46) vanishes as $n$ tends to infinity.

C. $C_{q}^{(\infty)}(W)$ is the Highest Achievable Rate for a Sufficiently Fast Decay of the Average Probability of Error

Let $\eta_{n}$ denote the smallest non-zero metric difference among all possible codewords and channel outputs; that is,

$$
\eta_{n}=\min _{x^{n}, \tilde{x}^{n}, y^{n}: q_{n}\left(\tilde{x}^{n}, y^{n}\right) \neq q_{n}\left(x^{n}, y^{n}\right)}\left|q_{n}\left(\tilde{x}^{n}, y^{n}\right)-q_{n}\left(x^{n}, y^{n}\right)\right|,
$$

where the minimization is over triplets of $n$-vectors in $\mathcal{X}^{n} \times \mathcal{X}^{n} \times \mathcal{Y}^{n}$. 
Note that if the set $\left\{x^{n}, \tilde{x}^{n}, y^{n}: q_{n}\left(\tilde{x}^{n}, y^{n}\right) \neq q_{n}\left(x^{n}, y^{n}\right)\right\}$ is non-empty, $\eta_{n}>0$ (with strict inequality), and if it is empty for any $n$, this means that the mismatch capacity of the channel is zero, since any two sequences $x^{n}, \tilde{x}^{n}$ are indistinguishable using the metric $q$ for every channel output.

The next theorem states that $C_{q}^{(\infty)}(W)$ is the highest achievable rate for sequences of codes whose average probability of error satisfies $\lim _{n \rightarrow \infty} \frac{1}{\eta_{n}} P_{e}\left(W^{n}, \mathcal{C}_{n}, q_{n}\right)=0$. This implies a soft converse as an important special case when the metric $q$ is rational; $C_{q}^{(\infty)}(W)$ is the highest achievable rate for code sequences whose average probability of error converges to zero faster than $O(1 / n)$.

Theorem 3. For a metric $q$ which satisfies the boundedness assumption (7), every code-sequence $\left\{\mathcal{C}_{n}\right\}_{n \in \mathbb{N}}$ for which $\lim _{n \rightarrow \infty} \frac{1}{\eta_{n}} P_{e}\left(W^{n}, \mathcal{C}_{n}, q_{n}\right)=0$ must satisfy

$$
R=\limsup _{n \rightarrow \infty} \frac{1}{n} \log \left|\mathcal{C}_{n}\right| \leq C_{q}^{(\infty)}(W) .
$$

In particular, if $q$ is a rational metric and $\lim _{n \rightarrow \infty} n \cdot P_{e}\left(W^{n}, \mathcal{C}_{n}, q_{n}\right)=0$, then $R \leq C_{q}^{(\infty)}(W)$.

Theorem 3 constitutes a step forward towards verifying whether Csiszár and Narayan's conjecture [18] holds; i.e., whether $C_{q}(W)=C_{q}^{(\infty)}(W)$. For rational metrics, it identifies $C_{q}^{(\infty)}(W)$ as the highest rate achievable with average probability of error which is $o(1 / n)$. The gap can be closed if one could show that the average probability of error vanishes exponentially fast (or at least as fast as $o(1 / n)$ ) at any rate below capacity. Since at rates below $C_{q}^{(\infty)}(W)$ exponential decay of the average probability of error is feasible [18], the following corollary can be stated.

Corollary 2. For a bounded rational metric $q, C_{q}(W)=C_{q}^{(\infty)}(W)$ iff for all $R<C_{q}(W)$ there exists a sequence of codebooks $\left\{\mathcal{C}_{n}\right\}_{n \in \mathbb{N}}$, such that $\liminf _{n \rightarrow \infty} \frac{1}{n} \log \left|\mathcal{C}_{n}\right| \geq R$ and $\liminf \operatorname{in}_{n \rightarrow \infty}-\frac{1}{n} \log P_{e}\left(W^{n}, \mathcal{C}_{n}, q_{n}\right)>0$.

We now prove Theorem 3 .

Proof. Fix a vanishing sequence $\tilde{\epsilon}_{n}, n \geq 1$. Let $\mathcal{P}_{U}\left(\tilde{\epsilon}_{n}\right)$ stand for the set of $P^{(n)} \in \mathcal{P}\left(\mathcal{X}^{n}\right)$ which are uniform over a subset $\mathcal{C}_{n}$ of $\mathcal{X}^{n}$ and such that $P\left(W^{(n)}, \mathcal{C}_{n}, q_{n}\right)=\tilde{\epsilon}_{n}$; i.e.,

$$
\begin{aligned}
& \mathcal{P}_{U}\left(\tilde{\epsilon}_{n}\right) \\
\triangleq & \left\{P^{(n)} \in \mathcal{P}\left(\mathcal{X}^{n}\right): P^{(n)}\left(\tilde{x}^{n}\right)=P^{(n)}\left(x^{n}\right) \text { if } P^{(n)}\left(x^{n}\right) \cdot P^{(n)}\left(\tilde{x}^{n}\right)>0, P\left(W^{(n)}, \mathcal{C}_{n}, q_{n}\right)=\tilde{\epsilon}_{n}\right\},
\end{aligned}
$$

where $\mathcal{C}_{n}$ stands for the support of $P^{(n)}$.

Let $P^{(n)} \in \mathcal{P}_{U}\left(\tilde{\epsilon}_{n}\right)$ be given, and denote by $\mathcal{C}_{n}$ the support set of $P^{(n)}$, which will be referred to as a codebook. Denote again $R_{n}=\frac{1}{n} \log \left|\mathcal{C}_{n}\right|$.

Let $\widetilde{\mathcal{E}}$ be the error event; that is,

$$
\widetilde{\mathcal{E}}=\left\{\left(x^{n}, y^{n}\right): q_{n}\left(x^{n}, y^{n}\right) \leq \max _{\tilde{x}^{n} \in \mathcal{C}_{n}: \tilde{x}^{n} \neq x^{n}} q_{n}\left(\tilde{x}^{n}, y^{n}\right)\right\},
$$

and note that $\operatorname{Pr}(\widetilde{\mathcal{E}})=P_{e}\left(W^{(n)}, \mathcal{C}_{n}, q_{n}\right)=\tilde{\epsilon}_{n}$. 
Next we invoke Lemma 1 with $\mathcal{A}=\widetilde{\mathcal{E}}$. Recall (15), we obtain

$$
\begin{aligned}
& \frac{1}{n} \sup _{s_{n} \geq 0, a\left(x^{n}\right) \geq 0} \mathbb{E}\left(\log \frac{e^{s_{n} q_{n}\left(X^{n}, Y^{n}\right)+a\left(X^{n}\right)}}{\sum_{\bar{x}^{n}} P^{(n)}\left(\bar{x}^{n}\right) e^{s_{n} q_{n}\left(\bar{x}^{n}, Y^{n}\right)+a\left(\bar{x}^{n}\right)}}\right) \\
\geq & \sup _{s_{n} \geq 0}-\operatorname{Pr}\{\widetilde{\mathcal{E}}\} \cdot \frac{s_{n} \cdot 2 B}{n}+\operatorname{Pr}\left\{\widetilde{\mathcal{E}}^{c}\right\} \cdot \mathbb{E}\left(\min \left\{R_{n}, \frac{s_{n}}{n} \cdot \min _{\bar{x}^{n} \in \mathcal{C}_{n}: \bar{x}^{n} \neq X^{n}}\left[q_{n}\left(X^{n}, Y^{n}\right)-q_{n}\left(\bar{x}^{n}, Y^{n}\right)\right]\right\} \mid \widetilde{\mathcal{E}}^{c}\right) \\
& \quad-\frac{1}{n} \log (2) \\
\geq & \sup _{s_{n} \geq 0}-\frac{\tilde{\epsilon}_{n} s_{n} \cdot 2 B}{n}+\left(1-\tilde{\epsilon}_{n}\right) \cdot \min \left\{R_{n}, \frac{s_{n} \eta_{n}}{n}\right\}-\frac{1}{n} \log 2
\end{aligned}
$$

where the first inequality follows from 16 by substituting $\mathcal{A} \Rightarrow \widetilde{\mathcal{E}}$ and the second in equality follows by definition of $\eta_{n}(48)$ and by the fact that under $\widetilde{\mathcal{E}}^{c}, q_{n}\left(X^{n}, Y^{n}\right)-\max _{\bar{x}^{n} \in \mathcal{C}_{n}: \bar{x}^{n} \neq X^{n}} q_{n}\left(\bar{x}^{n}, Y^{n}\right) \geq \eta_{n}$.

Finally we substitute $3 s_{n}=\frac{n R_{n}}{\eta_{n}}$, and take the supremum over the sequences $\tilde{\epsilon}_{1}, \tilde{\epsilon}_{2}, \ldots$ which satisfy $\lim _{n \rightarrow \infty} \frac{\tilde{\epsilon}_{n}}{\eta_{n}}=$ 0 , this yields

$$
C_{q}^{(\infty)}(W) \geq \sup _{\substack{\left\{\tilde{\epsilon}_{n}\right\}_{n \geq 1}: \tilde{\epsilon}_{n} \geq 0, \lim _{n \rightarrow \infty} \frac{\epsilon_{n}}{\eta_{n}}=0}} \limsup _{n \rightarrow \infty} \max _{P^{(n)} \in \mathcal{P}_{U}\left(\tilde{\epsilon}_{n}\right)}-\frac{\tilde{\epsilon}_{n} R_{n} \cdot 2 B}{\eta_{n}}+R_{n} .
$$

Now, the r.h.s. of 53 is nothing but the supremum of the rates for which $\lim _{n \rightarrow \infty} \frac{\tilde{\epsilon}_{n}}{\eta_{n}}=0$ and this concludes the proof of the first statement of Theorem 3

To prove the second statement of the theorem pertaining to rational metrics it suffices to prove that for a rational metric $q$ with denominator $D$ one has $\eta_{n} \geq \frac{1}{n D}$ as the following lemma states.

Lemma 4. If $q$ is a rational metric with denominator $D$, then

$$
\max _{\tilde{x}^{n}, x^{n}, y^{n}: q_{n}\left(\tilde{x}^{n}, y^{n}\right) \neq q_{n}\left(x^{n}, y^{n}\right)}\left|q_{n}\left(\tilde{x}^{n}, y^{n}\right)-q_{n}\left(x^{n}, y^{n}\right)\right| \geq \frac{1}{n D} .
$$

Proof. Since the metric is rational with denominator $D$, we can write

$$
q_{n}\left(\tilde{x}^{n}, y^{n}\right)=\frac{1}{n} \sum_{i=1}^{n} \frac{a_{i}}{D} \quad ; \quad q_{n}\left(x^{n}, y^{n}\right)=\frac{1}{n} \sum_{i=1}^{n} \frac{b_{i}}{D},
$$

where $a_{i}, b_{i}, i=1, \ldots, n$, are integers. Thus, if $q_{n}\left(\tilde{x}^{n}, y^{n}\right) \neq q_{n}\left(x^{n}, y^{n}\right)$, assume w.l.o.g. that $q_{n}\left(\tilde{x}^{n}, y^{n}\right)>$ $q_{n}\left(x^{n}, y^{n}\right)$, and we obtain

$$
\sum_{i=1}^{n} a_{i}>\sum_{i=1}^{n} b_{i}
$$

Consequently, we deduce that

$$
\sum_{i=1}^{n} a_{i} \geq 1+\sum_{i=1}^{n} b_{i}
$$

which yields

$$
\sum_{i=1}^{n} \frac{a_{i}}{n D} \geq \frac{1}{n D}+\sum_{i=1}^{n} \frac{b_{i}}{n D}
$$

and the desired result follows.

This concludes the proof of Theorem 3

${ }^{3}$ We choose $s_{n}=\frac{n R_{n}}{\eta_{n}}$ for the term $\min \left\{R_{n}, \frac{s_{n} \delta}{n}\right\}$ to yield $R_{n}$ which is our desired lower bound. 


\section{A MAX-Min UPPER BOUnd}

We next present an upper bound on $C_{q}(W)$ which differs from the lower bound $C_{q}^{(\infty)}(W)$ by the sets over which the inner minimizations are performed.

Theorem 4. The mismatch capacity of the DMC $W$ with an additive metric $q$ satisfies

$$
C_{q}(W) \leq \liminf _{n \rightarrow \infty} \max _{P_{X^{n}}} \min _{\substack{P_{\tilde{Y} n} \mid X^{n}: \\ P_{\tilde{Y} n}, q_{n}\left(X^{n}, \tilde{Y}^{n}\right)=P_{Y^{n}, q_{n}\left(X^{n}, Y^{n}\right)}}} \frac{1}{n} I\left(X^{n} ; \tilde{Y}^{n}\right),
$$

where $P_{\widetilde{Y}^{n}, q_{n}\left(X^{n}, \widetilde{Y}^{n}\right)}$ is the joint distribution of $\widetilde{Y}^{n}$ and the (random) metric value $q_{n}\left(X^{n}, \widetilde{Y}^{n}\right)$ at the output of the channel $P_{\widetilde{Y}^{n} \mid X^{n}}$, and $P_{Y^{n}, q_{n}\left(X^{n}, Y^{n}\right)}$ is defined as for the channel $W^{n}$.

Proof. The proof is similar to the proof of [21, Theorem 5].

Let

$$
\Phi_{q_{n}} \triangleq \operatorname{Pr}\left\{q_{n}\left(\tilde{X}^{n}, Y^{n}\right) \geq q_{n}\left(X^{n}, Y^{n}\right) \mid X^{n}, Y^{n}\right\}
$$

where $X^{n}$ and $Y^{n}$ are the input and output channel vectors, respectively, and $\tilde{X}^{n}$ is independent of $\left(X^{n}, Y^{n}\right)$ and is distributed identically to $X^{n}$.

It was noted in [21] that for every codebook $\mathcal{C}_{n}$, one has $P_{e}\left(W^{n}, \mathcal{C}_{n}, q_{n}\right)=P_{e}\left(\widetilde{W}^{(n)}, \mathcal{C}_{n}, q_{n}\right)$ for every channel $\widetilde{W}^{(n)}$ whose output $\widetilde{Y}^{n}$ shares the same joint law with $q_{n}\left(X^{n}, \widetilde{Y}^{n}\right)$ as that of $\left(Y^{n}, q_{n}\left(X^{n}, Y^{n}\right)\right)$. This is evident from the expression

$$
\begin{aligned}
& P_{e}\left(W^{(n)}, \mathcal{C}_{n}, q_{n}\right) \\
= & \operatorname{Pr}\left\{-\frac{1}{n} \log \Phi_{q_{n}}<\frac{1}{n} \log M_{n}\right\},
\end{aligned}
$$

which was derived in [21, Lemma 1].

The r.h.s. of 61 is identical for both channels $W^{(n)}$ and $\widetilde{W}^{(n)}$. Consequently, if $P_{\widetilde{Y}^{n}, q_{n}\left(X^{n}, \widetilde{Y}^{n}\right)}=P_{Y^{n}, q_{n}\left(X^{n}, Y^{n}\right)}$ for all $n$, the capacity of the channel $W^{n}$ is equal to that of the channel $\left\{\widetilde{W}^{(n)}\right\}_{n \in \mathbb{N}}$, whose mismatch capacity can in turn, be bounded essentially by the normalized mutual information $\frac{1}{n} I\left(X^{n} ; \widetilde{Y}^{n}\right)$ yielding 59 .

\section{CONCLUSION}

In this work we considered a DMC with decoders that employ a mismatched metric. Finding a single-letter expression for the mismatch capacity of the DMC remains an open problem. Nevertheless, inspired by Csiszár and Narayan's conjecture which states that the "product-space" improvement of the random coding lower bound on the mismatch capacity $C_{q}^{(\infty)}(W)$ is the channel capacity, we studied this quantity. The significance of Csiszár and Narayan's conjecture is threefold because if true, it implies that: (a) one can approach capacity by employing random coding schemes with product space input alphabets of increasing dimension, (b) the mismatch capacity is equal to the threshold capacity. (c) exponential decay of the average probability of error at rate $R-\epsilon$ for all $\epsilon>0$ is feasible. 
Several properties of $C_{q}^{(\infty)}(W)$ were proved in this paper. The first property is that $C_{q}^{(\infty)}(W)$ is equal to the mismatched capacity with a constant margin level. It is known [1], [2] that $C_{q}^{(\infty)}(W)$ is upper bounded by the constant threshold capacity. In this paper we derived the opposite inequality for the case of $P$-constant compositions codes. We proved that the highest achievable rate with a constant margin decoder is equal to the highest achievable rate with a constant threshold decoder for sequences of $P$-constant compositions codes. Consequently, if such sequences of codes are used with constant threshold decoding, the highest achievable rate is upper bounded by $C_{q}^{(\infty)}(W)$. The second property states that if the average probability of error of a sequence of codebooks, $\epsilon_{n}$, converges to zero sufficiently fast, in particular if $\epsilon_{n} / \eta_{n}$ vanishes (where $\eta_{n}$ is the minimal non-zero difference among all possible metric values between sequences of length $n$ ), the rate of the code-sequence is upper bounded by $C_{q}^{(\infty)}(W)$. Consequently, in particular, we have shown that if $q$ is a bounded rational metric $\eta_{n}=O(1 / n)$, and thus in this case, if the average probability of error converges to zero faster than $O(1 / n)$, then $R \leq C_{q}^{(\infty)}(W)$. It therefore can be inferred that in this case if a sequence of codes of rate $R$ is known to achieve an average probability of error which is $o(1 / n)$, there exists a sequence of codes operating at a rate arbitrarily close to $R$ with an average probability of error that vanishes exponentially fast. It would be an interesting problem to specify a lower bound on the convergence rate of $\eta_{n}$ to zero as $n$ tends to infinity for non rational metrics, which will lead to more general result.

Although we could not verify whether Csiszár and Narayan's conjecture holds, the results obtained in this paper increase our understanding of the quantity $C_{q}^{(\infty)}(W)$ and constitute a step forward towards verifying whether the conjecture holds.

We concluded by deriving a max-min multi-letter upper bound on the mismatch capacity $C_{q}(W)$ which bears some resemblance to $C_{q}^{(\infty)}(W)$. While the upper bound does not match the known lower bounds, the proof technique may shed some light on the mismatch problem.

\section{ACKNOWLEDGEMENT}

We would like to thank the anonymous reviewers for their invaluable comments and suggestions, which led to a significant simplification of the proofs and contributed to the conciseness thereof.

\section{APPENDIX}

\section{A. Proof of Lemma 2}

Recall the definitions of $n_{x}, A_{x}$ (44) and recall (45). As mentioned above, $n_{x}$ is a deterministic quantity since all $x^{n}$ 's lie in the same type-class. This implies that $q_{n}\left(X^{n}, Y^{n}\right)$ is the average of $|\mathcal{X}|$ independent random variables $A_{x}, x \in \mathcal{X}$, each of which is the average of at least $n \cdot \min _{x \in \mathcal{X}} \hat{P}_{n}(x)$ i.i.d, random variables. By assumption, $P(x)>$ $0, \forall x \in \mathcal{X}$, which implies that there exists $\zeta>0$ such that $A_{x}$ is a average of at least $n \zeta$ i.i.d. random variables, and since the exists at least one symbol $x^{*}$ which satisfies $\max _{y, y^{\prime} \in \mathcal{Y}} W\left(y \mid x^{*}\right) q\left(x^{*}, y\right)-W\left(y^{\prime} \mid x^{*}\right) q\left(x^{*}, y^{\prime}\right) \neq 0$, at 
least one of the random variables $A_{x}, x \in \mathcal{X}$ is the average of $n \zeta$ i.i.d. random variables having a strictly positive variance. Let $\mathcal{X}^{\prime}$ be the set of $x$ 's such that the variance of $A_{x}$ is positive. We have for an arbitrary $x^{n} \in \mathcal{C}_{n}$,

$$
\begin{aligned}
& \operatorname{Pr}\left\{q_{n}\left(X^{n}, Y^{n}\right)<\mathbb{E}_{\hat{P}_{n} \times W}(q(X, Y))-k n^{-1 / 2}\right\} \\
& \stackrel{(a)}{=} \operatorname{Pr}\left\{q_{n}\left(X^{n}, Y^{n}\right)<\mathbb{E}_{\hat{P}_{n} \times W}(q(X, Y))-k n^{-1 / 2} \mid X^{n}=x^{n}\right\} \\
& \stackrel{(b)}{\geq} \operatorname{Pr}\left\{\bigcap_{x \in \mathcal{X}^{\prime}}\left\{\frac{1}{n_{x}} \sum_{i=1}^{n} 1\left\{X_{i}=x\right\} q\left(x, Y_{i}\right)<\mathbb{E}_{W(\cdot \mid x)}(q(x, Y))-k n^{-1 / 2}\right\} \mid X^{n}=x^{n}\right\} \\
&= \prod_{x \in \mathcal{X}^{\prime}} \operatorname{Pr}\left\{\frac{1}{n_{x}} \sum_{i=1}^{n} 1\left\{X_{i}=x\right\} q\left(x, Y_{i}\right)<\mathbb{E}_{W(\cdot \mid x)}(q(x, Y))-k n^{-1 / 2} \mid X^{n}=x^{n}\right\} .
\end{aligned}
$$

where $(a)$ follows since $\mathcal{C}_{n}$ is a constant composition, and $(b)$ follows since if for all $x, a_{x} \leq b_{x}$ then for any p.m.f. $\mu \in \mathcal{P}(\mathcal{X}), \frac{1}{|\mathcal{X}|} \sum_{x=1}^{|\mathcal{X}|} a_{x} \leq \frac{1}{|\mathcal{X}|} \sum_{x=1}^{|\mathcal{X}|} b_{x}$

Now, given $X^{n}=x^{n}, A_{x}$ is the average of $n_{x}$ positive-variance i.i.d. random variables with expectation $\mathbb{E}_{W(\cdot \mid x)}(q(x, Y))$.

For $\ell$ i.i.d. random variables $Z_{i}$ whose expectation and variance are $\mu$ and $\sigma^{2}$, respectively, we have from the CLT

$$
\begin{aligned}
& \lim _{\ell \rightarrow \infty} \operatorname{Pr}\left\{\frac{1}{\ell} \sum_{i=1}^{\ell} Z_{i} \leq \mu-k(\ell \cdot c)^{-1 / 2}\right\} \\
= & \lim _{\ell \rightarrow \infty} \operatorname{Pr}\left\{\frac{1}{\sqrt{\ell} \sigma} \sum_{i=1}^{\ell}\left(Z_{i}-\mu\right) \leq-k c^{-1 / 2} / \sigma\right\} \\
= & \Phi\left(-k c^{-1 / 2} / \sigma\right)>0,
\end{aligned}
$$

where $\Phi(\cdot)$ is the cumulative distribution function of the standard Gaussian distribution.

Applying this to 62 we obtain

$$
\begin{aligned}
& \operatorname{Pr}\left\{q_{n}\left(X^{n}, Y^{n}\right)<\mathbb{E}_{\hat{P}_{n} \times W}(q(X, Y))-k n^{-1 / 2} \mid X^{n}=x^{n}\right\} \\
\geq & \left(\Phi\left(-k \zeta^{-1 / 2} / \sigma\right)\right)^{\left|\mathcal{X}^{\prime}\right|}>0,
\end{aligned}
$$

where $\sigma^{2}$ is the positive variance mentioned above.

Therefore,

$$
\lim _{n \rightarrow \infty} \operatorname{Pr}\left\{q_{n}\left(X^{n}, Y^{n}\right)<\mathbb{E}_{\hat{P}_{n} \times W}(q(X, Y))-k n^{-1 / 2}\right\}>0,
$$

which concludes the proof of Lemma 2

\section{B. Proof of Lemma 3}

First, note that

$$
\begin{aligned}
& P_{\text {margin }}\left(W^{(n)}, \mathcal{C}_{n}, q_{n}, \epsilon\right) \\
= & \operatorname{Pr}\left\{\max _{x^{n} \in \mathcal{C}_{n}: x^{n} \neq X^{n}} q_{n}\left(x^{n}, Y^{n}\right) \geq q_{n}\left(X^{n}, Y^{n}\right)-\epsilon\right\} .
\end{aligned}
$$


Next, define the events $\mathcal{A}_{1}=\left\{\max _{x^{n} \in \mathcal{C}_{n}: x^{n} \neq X^{n}} q_{n}\left(x^{n}, Y^{n}\right) \geq \tau\right\}$ and $\mathcal{A}_{2}=\left\{q_{n}\left(X^{n}, Y^{n}\right)<\tau\right\}$, whose union is the error event of the threshold decoder; i.e., $P_{\text {thresh }}\left(W^{(n)}, \mathcal{C}_{n},\left(q_{n}, \tau\right)\right)=\operatorname{Pr}\left\{\mathcal{A}_{1} \cup \mathcal{A}_{2}\right\}$. We have,

$$
\begin{aligned}
& \operatorname{Pr}\left\{\max _{x^{n} \in \mathcal{C}_{n}: x^{n} \neq X^{n},} q_{n}\left(x^{n}, Y^{n}\right) \geq q_{n}\left(X^{n}, Y^{n}\right)-\epsilon\right\} \\
= & \operatorname{Pr}\left\{\left\{\max _{x^{n} \in \mathcal{C}_{n}: x^{n} \neq X^{n}} q_{n}\left(x^{n}, Y^{n}\right) \geq q_{n}\left(X^{n}, Y^{n}\right)-\epsilon\right\} \cap\left(\mathcal{A}_{1} \cup \mathcal{A}_{2}\right)\right\} \\
& +\operatorname{Pr}\left\{\left\{\max _{x^{n} \in \mathcal{C}_{n}: x^{n} \neq X^{n}} q_{n}\left(x^{n}, Y^{n}\right) \geq q_{n}\left(X^{n}, Y^{n}\right)-\epsilon\right\} \cap\left(\mathcal{A}_{1} \cup \mathcal{A}_{2}\right)^{c}\right\} \\
\leq & \operatorname{Pr}\left\{\mathcal{A}_{1} \cup \mathcal{A}_{2}\right\} \\
& +\operatorname{Pr}\left\{\left\{\max _{x^{n} \in \mathcal{C}_{n}: x^{n} \neq X^{n}} q_{n}\left(x^{n}, Y^{n}\right) \geq q_{n}\left(X^{n}, Y^{n}\right)-\epsilon\right\} \cap \mathcal{A}_{1}^{c}\right\} \\
\leq & P_{\text {thresh }}\left(W^{(n)}, \mathcal{C}_{n},\left(q_{n}, \tau\right)\right)+\operatorname{Pr}\left\{q_{n}\left(X^{n}, Y^{n}\right) \leq \tau+\epsilon\right\},
\end{aligned}
$$

where the last step follows since

$$
\begin{aligned}
& \left\{\exists x^{n} \in \mathcal{C}_{n}: x^{n} \neq X^{n}, q_{n}\left(x^{n}, Y^{n}\right) \geq q_{n}\left(X^{n}, Y^{n}\right)-\epsilon\right\} \cap \mathcal{A}_{1}^{c} \\
= & \left\{\max _{x^{n} \in \mathcal{C}_{n}: x^{n} \neq X^{n}} q_{n}\left(x^{n}, Y^{n}\right) \geq q_{n}\left(X^{n}, Y^{n}\right)-\epsilon\right\} \cap\left\{\max _{x^{n} \in \mathcal{C}_{n}: x^{n} \neq X^{n}} q_{n}\left(x^{n}, Y^{n}\right)<\tau\right\} \\
\subseteq & \left\{q_{n}\left(X^{n}, Y^{n}\right) \leq \tau+\epsilon\right\},
\end{aligned}
$$

proving Lemma 3.

\section{REFERENCES}

[1] I. Csiszár and J. Körner, "Graph decomposition: A new key to coding theorems," IEEE Trans. Inf. Theory, vol. 27, no. 1, pp. 5-12, 1981.

[2] J. Hui, "Fundamental issues of multiple accessing," PhD dissertation, MIT, 1983.

[3] A. Lapidoth, "Mismatched decoding and the multiple-access channel," IEEE Trans. Inf. Theory, vol. 42, no. 5, pp. 1439-1452, Sept. 1996.

[4] A. Somekh-Baruch, "On achievable rates and error exponents for channels with mismatched decoding," IEEE Trans. Inf. Theory, vol. 61, no. 2, pp. 727-740, Feb 2015.

[5] A. Somekh-Baruch, "On coding schemes for channels with mismatched decoding," in Proc. Int. Symp. Information Theory, ISIT, Istanbul,Turkey, 2013.

[6] J. Scarlett, A. Martinez, and A. Guillén i Fàbregas, "Superposition codes for mismatched decoding," in Proc. Int. Symp. Information Theory, ISIT, Istanbul,Turkey, 2013, pp. 81-85.

[7] —_, "Multiuser coding techniques for mismatched decoding," arXiv:1311.6635 [cs.IT], Nov. 2013.

[8] V. Balakirsky, "Coding theorem for discrete memoryless channels with given decision rule," in Algebraic Coding, ser. Lecture Notes in Computer Science, G. Cohen, A. Lobstein, G. Zémor, and S. Litsyn, Eds. Springer Berlin Heidelberg, 1992, vol. 573, pp. 142-150. [Online]. Available: http://dx.doi.org/10.1007/BFb0034351

[9] G. Kaplan and S. Shamai, "Information rates and error exponents of compound channels with application to antipodal signaling in a fading environment," AEU. Archiv für Elektronik und Übertragungstechnik, vol. 47, no. 4, pp. 228-239, 1993.

[10] N. Merhav, G. Kaplan, A. Lapidoth, and S. Shamai (Shitz), "On information rates for mismatched decoders," IEEE Trans. Inf. Theory, vol. 40, no. 6, pp. 1953-1967, Nov. 1994.

[11] A. Lapidoth, "Nearest neighbor decoding for additive non-Gaussian noise channels," IEEE Trans. Inf. Theory, vol. 42, no. 5, pp. 1520-1529, Sept. 1996.

[12] A. Ganti, A. Lapidoth, and I. Telatar, "Mismatched decoding revisited: general alphabets, channels with memory, and the wide-band limit," IEEE Trans. Inf. Theory, vol. 46, no. 7, pp. 2315-2328, Nov. 2000.

[13] S. Shamai and I. Sason, "Variations on the Gallager bounds, connections, and applications," IEEE Trans. Inf. Theory, vol. 48, no. 12, pp. 3029-3051, 2002. 
[14] J. Scarlett and A. Guillén i Fàbregas, "An achievable error exponent for the mismatched multiple-access channel," in 50th Annual Allerton Conference on Communication, Control, and Computing (Allerton), October 2012, pp. 1975-1982.

[15] J. Scarlett, A. Martinez, and A. Guillén i Fàbregas, "Mismatched decoding: Error exponents, second-order rates and saddlepoint approximations," IEEE Trans. Inf. Theory, vol. 60, no. 5, pp. 2647-2666, May 2014.

[16] — _ "Ensemble-tight error exponents for mismatched decoders," in 50th Annual Allerton Conference on Communication, Control, and Computing (Allerton), 2012, pp. 1951-1958.

[17] J. Scarlett, L. Peng, N. Merhav, A. Martinez, and A. Guillén i Fàbregas, "Expurgated random-coding ensembles: Exponents, refinements, and connections," IEEE Trans. Inf. Theory, vol. 60, no. 8, pp. 4449-4462, Aug 2014.

[18] I. Csiszár and P. Narayan, "Channel capacity for a given decoding metric," IEEE Trans. Inf. Theory, vol. 41, no. 1, pp. 35-43, Jan. 1995.

[19] V. B. Balakirsky, "A converse coding theorem for mismatched decoding at the output of binary-input memoryless channels," IEEE Trans. Inf. Theory, vol. 41, no. 6, pp. 1889-1902, Nov. 1995.

[20] J. Scarlett, A. Somekh-Baruch, A. Martinez, and A. Guillén i Fàbregas, "A counter-example to the mismatched decoding converse for binary-input discrete memoryless channels," IEEE Trans. Inf. Theory, vol. 61, no. 10, pp. 5387-5395, Oct 2015.

[21] A. Somekh-Baruch, "A general formula for the mismatch capacity," IEEE Trans. Inf. Theory, vol. 61, no. 9, pp. 4554-4568, Sept 2015.

[22] S. Verdú and T. S. Han, "A general formula for channel capacity," IEEE Trans. Inf. Theory, vol. 40, no. 4, pp. 1147-1157, July 1994.

[23] A. Lapidoth and J. Ziv, "On the universality of the LZ-based decoding algorithm," IEEE Transactions on Information Theory, vol. 44, no. 5, pp. 1746-1755, Sep 1998.

[24] T. Hashimoto, "Composite scheme LR+TH for decoding with erasures and its effective equivalence to Forney's rule," IEEE Transactions on Information Theory, vol. 45, no. 1, pp. 78-93, Jan 1999.

[25] A. Somekh-Baruch, "Multi-letter converse bounds for the mismatched discrete memoryless channel with an additive metric," in Information Theory (ISIT), 2015 IEEE International Symposium on, June 2015, pp. 531-535. 